\title{
Optimal foraging in patchy turbulent environments
}

\author{
Jonathan W. Pitchford ${ }^{1, *}$, Alex James ${ }^{2}$, John Brindley ${ }^{3}$ \\ ${ }^{1}$ Department of Biology, University of York, PO Box 373, York YO10 5YW, United Kingdom \\ ${ }^{2}$ Department of Science and Mathematics, Sheffield Hallam University, Sheffield S1 1WB, United Kingdom \\ ${ }^{3}$ Department of Applied Mathematics, University of Leeds, Leeds LS2 9JT, United Kingdom
}

\begin{abstract}
The problem of what strategy a predator should adopt when foraging in a turbulent and spatially patchy environment is investigated using simple mathematical models. The study was motivated by the need to understand the behaviour of marine fish larvae searching for copepod prey. It is demonstrated that optimal swimming speed should decrease with increasing turbulence, and that in a patchy turbulent environment it is best to concentrate swimming activity within patches of prey until a threshold of turbulence is exceeded and swimming ceases to be energetically favourable. If the predator is unaware of its global environment, or is only able to make foraging decisions based on temporally and spatially local knowledge, then its ability to forage in a near-optimal manner is severely reduced.
\end{abstract}

KEY WORDS: Optimal foraging $\cdot$ Turbulence $\cdot$ Patchiness $\cdot$ Fish larvae $\cdot$ Individual-based model

\section{INTRODUCTION}

Classical theories concerning how an organism should choose to search for its food typically relate to a predator that is able to make behavioural choices (Charnov 1976, Stephens \& Krebs 1986, Houston \& McNamara 2001). Such a predator must be aware of its global environment and must be able to alter its circumstances within that environment. Consider, for example, a bird foraging in an environment in which small amounts of food are distributed in discrete patches. One foraging strategy is simply to remain in a patch until all the food therein is consumed and then to fly to another patch. A better strategy might be to remain in the first patch until the rate of finding food declines to a (non-zero) threshold before investing energy in flying to another patch. In order to effect the former strategy the bird must sense whether or not its current patch contains food, and be able to remain within, and move between, patches at will. For the second strategy, the bird needs to know, in addition, its current consumption rate and how this might change were it to move to another patch (Ollason 1980).

The aim here is to investigate how theories of optimal foraging behaviour change when considering predators whose knowledge of the environment is temporally and spatially localised, and who are unable directly to influence their position within the environment. Essentially, the predators concerned can be thought of as being 'small' and 'naïve' (a discussion of what constitutes smallness and naïvety will be developed in the paper). The motivation came from attempts to model the growth of fish larvae (Cushing \& Horwood 1994) in a turbulent ocean, where the copepod prey are patchily distributed (Pitchford \& Brindley 2001). In our case the larvae are 'small' in relation to typical turbulent velocities on the scales of spatial prey heterogeneity, and 'naïve' in that their foraging behaviour is thought to be governed by visual stimuli within a perceptive distance of around 1 body length. In the study of Pitchford \& Brindley (2001), which was principally concerned with understanding the population dynamics of recruitment into an adult population, it was argued that the mean rate at which an individual consumes prey is unaffected by prey patchiness (although increased patchiness leads to an increase in the variance of this rate). The model assumed that larvae swim at a constant speed, and encounter patches and prey stochastically as renewal processes (Beyer \& Nielsen 1996). Numerical simulations (Pitchford \& 
Brindley 2001, their Fig. 6) have shown that incorporating a small (and in our case rather artificial) change in larval behaviour within patches can lead to a large change in recruitment. Experimental work has shown that fish larvae can and do adapt their behaviour to changes in their environment (Døving et al. 1994, Gallego 1994, MacKenzie \& Kiørboe 1995, Munk 1995). These data are discussed more fully below. It is the purpose of this paper to elucidate which foraging strategies should be adopted by small and naïve foragers in turbulent and patchy environments, to ask whether such optimal behaviour is achievable in practice, and to compare the model predictions with experimental data. The mathematical modelling is deliberately kept as simple as possible in order to highlight the key processes at work.

We first introduce and develop the basic modelling framework, showing clearly the model's key assumptions and their mathematical consequences. This model is then used as a basis from which to investigate its suitability for optimal foraging strategies in different environments. Where possible an analytical approach is used and some general conclusions are drawn. When predators can respond to prey patches in a turbulent environment, numerical computations are necessary, the results of which are verified by comparison with explicit individual-based models (IBMs). Further results from IBM simulations are presented, this time with the predator's behaviour dictated only by its (temporal and spatial) local foraging history. The aim was to discover whether predators can ever achieve a near-optimal foraging strategy in a turbulent environment by employing only simple local rules, and the answer seems to be 'no'.

\section{BASIC MODEL}

The model developed here assumes that predatorpatch and predator-prey encounters occur as renewal processes (Cox 1962). The intensities of the temporally local Poisson point processes governing these encounters depend on both larval swimming speed and the turbulent fluid environment (Beyer \& Nielsen 1996, Pitchford \& Brindley 2001). Larvae swim at a constant speed, consuming a prey item if it falls within their limited field of vision (typically around 1 body length) (Sundby 1997). The larvae do not enter or leave patches by choice (strictly speaking, they are unaware they are ever in a patch) but by 'chance', either by swimming or by being advected by turbulence. It is assumed that no food exists outside the patches and that the density of larvae is insufficient to significantly deplete the concentration of food within a patch in the short term. The explicit assumptions relating to the behaviour of larvae are (1) each larva swims at a constant speed $v$ at all times when it is not encountering a prey item; (2) each larva is unaware of its environment outside its (small) perceptive distance, a hemisphere of radius $R$ centred at its eyes and pointing in the direction of swimming; (3) if a prey item enters the larva's perceptive field, the larva initiates an attack response, which takes a fixed time $\tau$, culminating in the prey being eaten and the larva resuming foraging at speed $v_{i}(4)$ the larvae do not graze down their prey at a rate high enough to cause any significant depletion in prey density during a visit to a patch.

The consequences of lifting certain of these restrictions will be investigated in subsections of this paper. If one defines $\alpha$ as the intensity (rate) of the Poisson process (Pp) with which a larva outside a patch encounters patches, $\beta$, to be the Pp intensity with which a larva in a patch leaves that patch, and $\gamma$ the Pp intensity with which a larva within a patch encounters prey, then under these assumptions one can assert, using turbulent encounter theory (Rothschild \& Osborn 1988, Sundby 1997, Pitchford \& Brindley 2001), that:

$$
\begin{gathered}
\alpha=\pi c_{\text {patch }} L^{2} \frac{\left(3 v^{2}+4 w_{L}^{2}\right)}{3\left(v^{2}+w_{L}^{2}\right) \frac{1}{2}} \\
\beta=\pi c_{\text {patch }} L^{2} \frac{\left(3 v^{2}+4 w_{L}^{2}\right)(1-V)}{3\left(v^{2}+w_{L}^{2}\right) \frac{1}{2} V} \\
\gamma=c_{\text {prey }} \pi R^{2} \frac{\left(3 v^{2}+4 w_{R}^{2}\right)}{3\left(v^{2}+w_{R}^{2}\right) \frac{1}{2}}
\end{gathered}
$$

Here, $w_{R}$ and $w_{L}$ are the root-mean-square turbulent velocities on the length scales $R$ and $L$ respectively, where $L$ is the size of a patch (Visser \& Mackenzie 1998). Assuming that a super-Kolmogorov scale model of turbulence can be applied, the turbulent velocities $w_{R}$ and $w_{L}$ can be calculated by:

$$
W_{a}=1.9(\varepsilon a)^{1 / 3}
$$

for length scales $a=R$ and $a=L$ (Hill et al. 1992, Sundby 1997); clearly each $w_{a}$ is an increasing function of turbulent intensity. The model does not take into account the fact that turbulence will tend to disperse prey patches; rather it is a pragmatic and tractable description of the observed turbulent and heterogeneous marine environment (Pitchford \& Brindley 2001 and references therein).

The prey are regarded as non-motile; the model can be extended to allow for randomly swimming prey (Sundby 1997), but such considerations are of minimal relevance in the context of fish larvae and copepod prey (Pitchford \& Brindley 2001). The local concentration of prey within patches is $C_{\text {prey }}$, while $c_{\text {patch }}$ represents the large-scale concentration of patches, i.e. the 
number of patches per unit volume. The constant $V$ in Eq. (2) refers to the fraction of the volume of the fluid that is taken up by prey patches; $0<V \leq 1$. (For spherical patches one can therefore assert that $V=3 / 4 \pi L^{3}$ $C_{\text {patch }}$. When predator swimming behaviour is constant within and outside patches, one can show that different-patch geometry, or allowing a range of patch sizes, has no qualitative effect on encounter rates [Pitchford \& Brindley 2001].) Using the above model, it can be shown (Pitchford \& Brindley 2001) that the mean rate at which predators encounter prey, $P$, is:

$$
P=\frac{\gamma \alpha}{\beta+\alpha(\gamma \tau+1)}
$$

This result assumes that each encounter always leads to a successful prey capture, which is reasonable at the moderate turbulent intensities considered here (Utne-Palm \& Stiansen 2002). The possibility that too much turbulence reduces the ability of the predators successfully to capture encountered prey (MacKenzie \& Kiørboe 2000) is therefore neglected; see Lewis \& Pedley (2001) for a comprehensive mathematical discussion of how to model the effect of turbulence on capture probability. Note that $P<1 / \tau$, since prey can never be successfully encountered faster than the predator can handle them. Dabrowski et al. (1988) estimate the gross energy contained in a copepod to be of the order $\mathrm{O}\left(10^{-2}\right) J$. However, $Q$ is the net increase in energy that a captured prey item confers upon the predator. Included within $Q$ are factors such as the efficiency of converting prey biomass into predator biomass, and thereafter into predator motion, which merit detailed modelling in themselves but which are not germane to the arguments presented here. These factors lead to $Q$ being substantially lower than the value given by Dabrowski et al. (1988). The prey are all regarded as identical in energy value, and any energetic costs of predatory behaviour during the duration $\tau$ of the attack are incorporated within $Q$.

Clearly, $P$ ought to increase with swimming speed $v$, so that the faster a predator swims the more prey it encounters. However, swimming faster involves a higher rate of energy expenditure. Define, therefore, $D(v)$ as the rate at which energy is used by a predator swimming at speed $v$. Here it is assumed that the predator expends energy to overcome viscous drag in the surrounding fluid, and Stokes' law is applied (Fuiman \& Batty 1997); for a steady swimmer this gives the function $D(v[t])$ as:

$$
D(v)=D_{0} v^{2}
$$

where $D_{0}=6 \pi \mu l, \mu$ is the viscosity of seawater, and $l$ is a typical length scale for a larva, taken here to be equal to its length. Dabrowski et al. $(1986,1988)$ and Weiser \& Kaufmann (1998) suggest an alternative empirical model based on the length of the larva and its swimming speed, and such approaches could easily be incorporated into the above model. In the region of interest, i.e. small fish larvae swimming at speeds of the order of 1 body length per second, the basic form of $D(v)$ is qualitatively identical, i.e. a positive increasing convex function of $v$, and one would not expect the results of the model to differ significantly. The model does not include a term for the basic metabolic rate. If this were taken as a constant with respect to swimming speed, its only effect would be to change the absolute value of the foraging efficiency. Clearly, any constant term will not affect the optimal values of the swimming speeds which are of interest here.

According to the above description, a swimming predator gains energy at an average rate, $P Q$, and loses energy at a rate $D_{0} V^{2}$. Since it swims for a proportion $(1-P \tau)$ of the time, its mean rate of acquiring energy when swimming at speed $v, F(v)$, is:

$$
F(v)=P Q-(1-P \tau) D_{0} v^{2}
$$

Henceforth $F(v)$ will be referred to as the predator's 'foraging efficiency'. The main thrust of this paper is to investigate the properties of the foraging efficiency, and of its extensions and generalisations. Note, in particular, that Eq. (7) will cease to be valid when one allows swimming speeds to change with time. It is also worth noting that since both the numerator and denominator of $P$ depend on $v$ and on turbulent velocities on different length scales, finding a value of $V$ which optimises $F(v)$ is not a trivial matter.

The definition of $F(v)$ in Eq. (7) is best thought of as the long-term average rate at which energy is acquired, i.e. if one measures the change in the predator's energy, $\Delta G$, over some finite (but possibly random) time $\Delta t$, then:

$$
F=\frac{E(\Delta G)}{E(\Delta t)}
$$

where $\mathrm{E}($.$) is the expectation operator. This is accepted$ as the most sensible measure of foraging efficiency (Charnov 1976, Stephens \& Krebs 1986, Houston \& McNamara 1999), and is appropriate for fish larvae foraging in a patchy environment, where one is typically concerned with larvae meeting a large number of patches and prey in the course of a day of fixed length. The alternative formulation for $F(v)$, where one considers the mean instantaneous rate of energy acquisition (Templeton \& Lawlor 1981), so that:

$$
E(F)=E\left(\frac{\Delta G}{\Delta t}\right)
$$

is less appropriate to a situation where energy is only acquired in discrete units, since this value varies enormously depending on the exact time interval $\Delta t$ cho- 
sen. Henceforth, therefore, only the former formulation (Eqs. $7 \& 8$ ) is considered, although individual-based simulations for the alternative form reveal qualitatively similar results. For the interested reader, Appendix 1 supplies a more detailed mathematical account of the arguments leading to Eq. (7).

\section{OPTIMAL FORAGING FOR OMNISCIENT PREDATORS}

Below, the optimal foraging problem is investigated for a situation in which the predator is aware of its global environment and of its current position within that environment, thus the results are derived from a mathematically idealised, rather than a biologically realistic, point of view. More biological realism is included subsequently, but the following results are important in defining general principles for foraging behaviour and as a comparison for more realistic modelling assumptions.

\section{Homogeneous prey distribution, constant swimming speed}

Prey patchiness can be removed from the model formulation simply by setting $\beta=0$ in Eqs. (5) \& (7), resulting in the simple expression:

$$
F(v)=\frac{Q \gamma-D_{0} v^{2}}{1+\gamma \tau}
$$

Fig. 1a illustrates this simplest of foraging behaviours diagrammatically. The turning points of Eq. (9) can be found in the usual way by differentiating with respect to $v$, setting the result equal to zero and solving for the critical values of $v$. This reveals that Eq. (9) has 2 non-negative turning points, the first at $v=0$ and the second at some positive value $v^{*}$. By examining the second derivative at these points it is seen that the turning point at $v=0$ is a minimum if:

$$
W_{R}<W_{R}^{*}=\frac{-3 D_{0}+\sqrt{9 D_{0}^{2}+16 D_{0} \pi^{2} R^{4} C_{\text {prey }}^{2} \tau Q}}{D_{0} \pi R^{2} C_{\text {prey }} \tau}
$$

If $w_{R}>W_{R}^{*}$ the turning point at $v=0$ becomes a maximum and the second turning point ceases to exist. This implies that for a low-turbulence environment there exists an optimum velocity $v^{*}>0$ which gives a maximum foraging efficiency. As the prey concentration increases, the foraging efficiency and this optimal swimming speed increase. As turbulence increases to $W_{R}^{*}$, this optimum velocity decreases to zero and the best strategy for the predator is to stop swimming and wait for the prey to be advected into range by turbu-
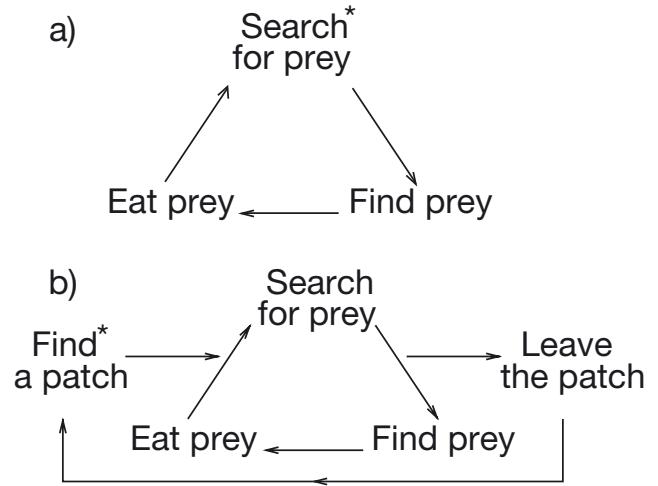

Fig. 1. Schematic diagrams showing patterns of behaviour followed by a predator. (a) Predator in homogeneous environment where same short pattern is repeated continuously. (b) Predator in patchy environment; here there is an additional cycle involving the predator searching for a patch. In both environments, from the predator's point of view it only ever does 1 thing - search for prey. *: starting point of each cycle as used in analysis

lence. This result is shown schematically in Fig. 2, and would appear to agree with the experimental results of MacKenzie \& Kiørboe (1995), as discussed in 'Discussion and conclusions'.

\section{Heterogeneous (patchy) prey, constant swimming speed}

Provided the predator swims at a constant speed at all times (other than during its prey encounters) Eq. (7) still holds. Moreover, it can be simplified by noticing that:

$$
\beta=\phi \alpha
$$

where the parameter $\phi={ }^{(1-V)} / V_{V}$ is the ratio of fluid outside patches to that within patches. Eq. (11) encapsulates the fact that under these assumptions the rate at which predators leave patches must, on average, balance the rate at which predators enter patches (Pitchford \& Brindley 2001). The expression for $F(v)$ then simplifies to:

$$
F(v)=\frac{\gamma Q-(1+\phi) D_{0} v^{2}}{1+\phi+\gamma \tau}
$$

The similarity with Eq. (9) is clear. Indeed, if one redefines the Pp intensity $\gamma$ as:

$$
\gamma^{*}=\gamma /(1+\phi)=\gamma V
$$

and performs some simple algebra, then Eq. (12) transforms directly into Eq. (9). From the definition of $\phi$ it becomes clear that $c_{\text {prey }}^{*}$ is simply the large-scale spatially averaged prey density. In other words, for a 'small and naïve' predator swimming at a constant 
speed, the optimal behaviour is unaffected by the details of prey patchiness; only the spatially averaged prey concentration is important. This result is in accordance with, and indeed is a consequence of, a result reported by Pitchford \& Brindley (2001) concerning mean predator-prey encounter rates.

These results suggest that the predator should never alter its behaviour in response to the perceived presence or absence of patches, a result which is at odds with observational evidence (see 'Discussion and conclusions'). It is the purpose of the following analysis, wherein the constant speed assumption is relaxed, to show that this result is not true in general, and that the predator can derive a huge advantage from being aware of, and responding to, its environment.

\section{Heterogeneous prey, variable swimming speed}

In this section it will be assumed that the predator is able to detect whether or not it is in a patch and is able to alter its swimming speed (but no other aspect of its behaviour) accordingly. Explicitly, let the predator swim at speed $V_{\text {out }}$ while outside patches and at $V_{\text {in }}$ within patches. In this case the Poisson process intensities $\alpha, \beta$ and $\gamma$ become:

$$
\begin{aligned}
& \alpha=\pi c_{\text {patch }} L^{2} \frac{\left(3 v_{\text {out }}^{2}+4 w_{L}^{2}\right)}{3\left(3 v_{\text {out }}^{2}+w_{L}^{2}\right) \frac{1}{2}} \\
& \beta=\pi c_{\text {patch }} L^{2} \phi \frac{\left(3 v_{\text {in }}^{2}+4 w_{L}^{2}\right)}{3\left(v_{\text {in }}^{2}+w_{L}^{2}\right) \frac{1}{2}} \\
& \gamma=c_{\text {prey }} \pi R^{2} \frac{\left(3 v_{\text {in }}^{2}+4 w_{R}^{2}\right)}{3\left(v_{\text {in }}^{2}+w_{R}^{2}\right) \frac{1}{2}}
\end{aligned}
$$

and the expression for $F(v)$ cannot be computed according to Eq. (7) since the costs for swimming within and outside patches are different. Consider, instead, a typical foraging bout as depicted in Fig. $1 b$; the larva starts outside a patch swimming at speed $V_{\text {out }}$. There is no prey outside patches, so the larva continues to swim and search for some finite time until it reaches a patch. Once within a patch the larva's behaviour is as in the homogeneous case, i.e. it searches for, finds, and attacks individual prey items, until it leaves the patch and the foraging cycle restarts.

Using basic Poisson process results, the mean time spent searching for a patch and the mean time spent swimming within a patch (excluding time spent capturing prey) are simply $1 / \alpha$ and $1 / \beta$ respectively. During a foraging bout a larva can expect to encounter $\gamma / \beta$ prey, so that the average rate of energy gain is:

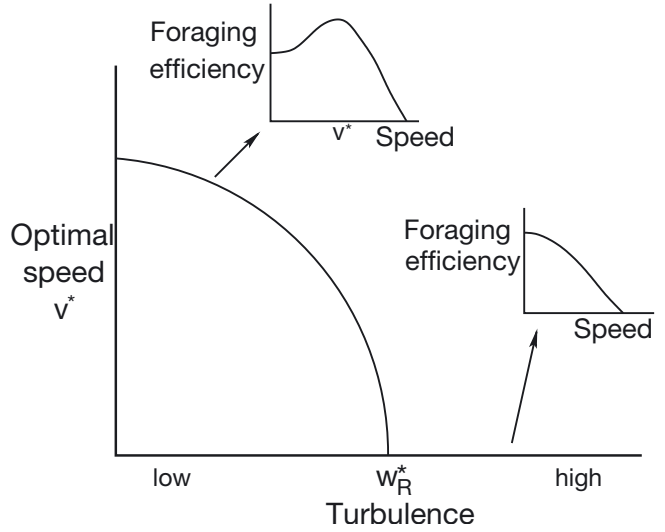

Fig. 2. Schematic diagram showing how optimal velocity changes as turbulence is varied for a larva swimming at constant speed in homogeneous prey environment. At low turbulence there is a local minimum of efficiency function $F(v)$ at $v=0$ and a maximum at $V=V^{*}$. As turbulence increases, $v^{*} \rightarrow 0$ as $w_{R} \rightarrow w_{R}^{*}$ and for $w_{R}>w_{R}^{*}$ optimal efficiency is achieved by the larvae not swimming

$$
F\left(v_{\text {in }}, v_{\text {out }}\right)=\frac{\frac{\gamma Q}{\beta}-D_{0}\left(\frac{v_{\text {out }}^{2}}{\alpha}+\frac{v_{\text {in }}^{2}}{\beta}\right)}{\frac{1}{\alpha}+\frac{1}{\beta}(1+\gamma \tau)}
$$

A more rigorous derivation of the same result can be obtained by generalising the approach given in Appendix 1. The authors have not found closed-form expressions maximising $F\left(V_{\text {in }}, V_{\text {out }}\right)$ in Eq. (17) with respect to its arguments for the general case. In the special case, where turbulent velocities on both the larval $(R)$ and patch $(L)$ length scales and the handling time $\tau$ are all zero, it can be shown that there is a unique maximum of $F\left(V_{\text {in }}, V_{\text {out }}\right)$ when:

$$
v_{\text {out }}=v_{\text {in }}=\frac{1}{2} \frac{C_{\text {prey }} R^{2} Q \pi}{D_{0}(1+\phi)}
$$

In other words, in a non-turbulent environment the optimal behaviour is always to swim at a constant speed. That this result is not true in general, but is an artifact of the assumption of zero turbulence, is demonstrated by the numerical examples below.

\section{Numerical examples: herring larvae foraging for copepods}

The following numerical simulations use the parameters summarised in Table 1, which describe a young herring larva of $5 \mathrm{~mm}$ in length (roughly $2 \mathrm{wk}$ posthatching). Parameters reflect typical conditions in the North Sea during a spring bloom, with a prey-patch length scale of $10 \mathrm{~m}$, these patches occupying $25 \%$ of 
Table 1. Standard parameter values used in numerical simulations

\begin{tabular}{|lccl|}
\hline Quantity & Symbol & Value & Source \\
\hline Concentration of patches & $C_{\text {patch }}$ & $0.75 \mathrm{~V} /\left(\pi \mathrm{L}^{3}\right)$ patches m $\mathrm{m}^{-3}$ & Pitchford \& Brindley (2001) \\
Concentration of prey in patches & $\mathrm{C}_{\text {prey }}$ & $1.5 \times 10^{4} \mathrm{copepods} \mathrm{m}^{-3}$ & Pitchford \& Brindley (2001) \\
Swimming cost coefficient & $D_{0}$ & $6 \pi \mu L \mathrm{~kg} \mathrm{~s}^{-1}$ & Fuiman \& Batty (1997) \\
Larval length & $I$ & $0.005 \mathrm{~m}$ & Munk (1995) \\
Patch length & $L$ & $10 \mathrm{~m}$ & Pitchford \& Brindley (2001) \\
Dynamic viscosity of seawater & $\mu$ & $1.5 \times 10^{-3} \mathrm{~kg} \mathrm{~ms}^{-1}$ & Fuiman \& Batty (1997) \\
Energy gained by eating 1 copepod & $Q$ & $1 \times 10^{-4} \mathrm{~J}$ & Dabrowski et al. (1988) \\
Larval perceptive distance & $R$ & $0.751 \mathrm{~m}$ & Sundby (1997) \\
Time taken to pursue and eat a copepod & $\tau$ & $2 \mathrm{~s}$ & MacKenzie \& Kiørboe (2000) \\
Fraction of volume occupied by prey & $V$ & 0.25 & Pitchford \& Brindley (2001) \\
Turbulent velocity at patch-length scale & $w_{L}$ & $1.9\left(7.4 \times 10^{8} L\right)^{1 / 3} \mathrm{~m} \mathrm{~s}^{-1}$ & MacKenzie \& Kiørboe (1995) \\
Turbulent velocity at larval-length scale & $w_{R}$ & $1.9\left(7.4 \times 10^{-8} R\right)^{1 / 3} \mathrm{~m} \mathrm{~s}^{-1}$ & MacKenzie \& Kiørboe (1995) \\
\hline
\end{tabular}

the fluid volume. However, due to uncertainty in parameter estimation, the value of these simulations is in demonstrating the model's basic validity, and in elucidating its main qualitative features. An advantage of the simple way in which the model is developed is that the parameters relating to individual behaviour and to physical processes can easily be adapted to other more specific problems.

Fig. 3 presents results from 2 complementary simulation methods; firstly by simply plotting foraging efficiencies given by Eq. (9), and secondly by direct simulation of 30000 foraging cycles of an individual larvae, encountering prey (and, in later scenarios, patches) as Poisson processes according to Eqs. (1) to (3). The mean foraging efficiency resulting from these IBMs is plotted. The agreement between the 2 approaches gives confidence in the theoretical development of the

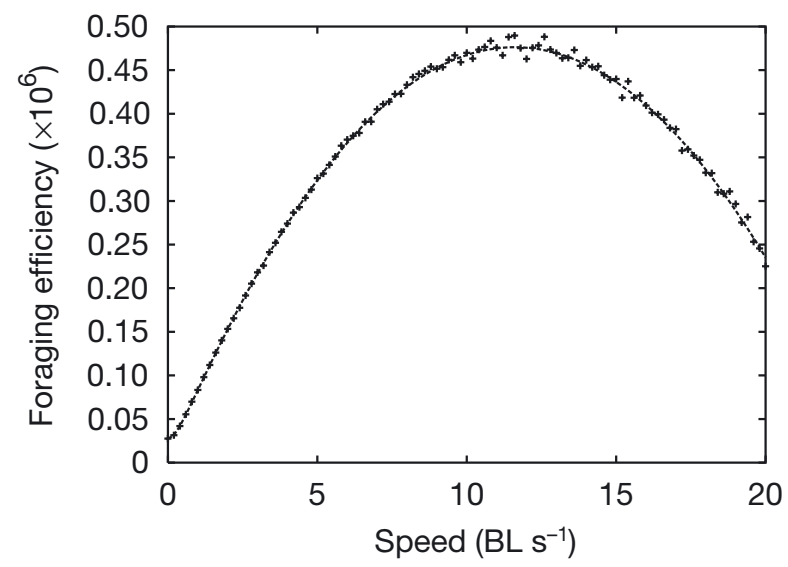

Fig. 3. Foraging efficiency in homogeneous environment. Line shows foraging efficiency function for different speeds, crosses show mean value of individual behaviour model calculated over 30000 cycles. All parameters are as in Table 1, and it is clear that this is within the low turbulence regime and optimum behaviour is to swim at around 12 body lengths $\mathrm{s}^{-1}$ model, and this is important for the subsequent modelling of larvae following behavioural rules, where closed-form expressions for foraging efficiency are not available. The same IBM simulation approach is used in Fig. 4 (and in the simulations of the behavioural rules in Figs. 8, 9 \& 10).

Fig. 3 shows the results of numerical simulations for a fish larva swimming in a homogeneous environment. The results show that the expected values of the foraging efficiency (dotted line in Fig. 3) derived from Eq. (9) match well with the individual-based simulations (crosses in Fig. 3). It is clear, with reference to Fig. 2, that this is a region of low turbulence, where the optimum behaviour for the larva is to swim rather than simply to wait for turbulence to bring it its food. The optimal speed is reasonable at around 12 body lengths $\mathrm{s}^{-1}\left(\mathrm{BL} \mathrm{s}^{-1}\right)$.

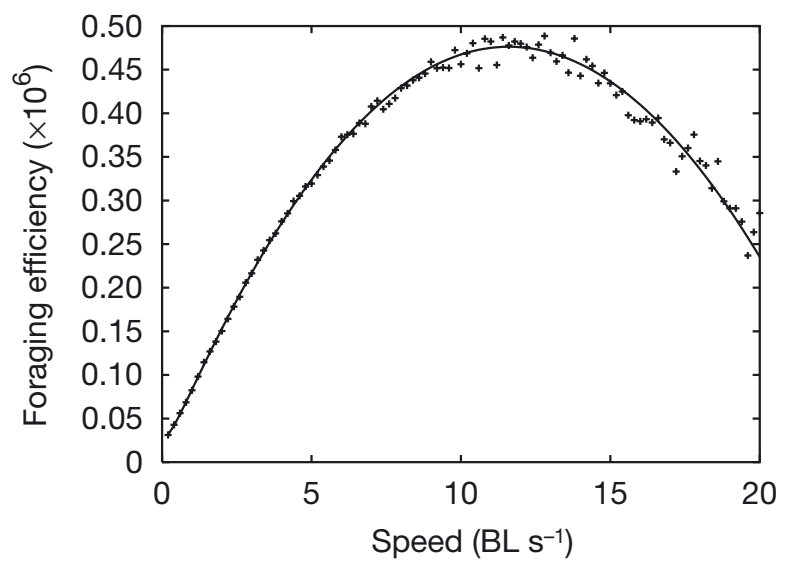

Fig. 4. Foraging efficiency in patchy environment with constant swimming speed. Line shows foraging efficiency function for different speeds, crosses show mean value of individual behaviour model calculated over 10000 cycles. These results are identical to those in Fig. 3 confirming result of Eqs. (11) to (13) 


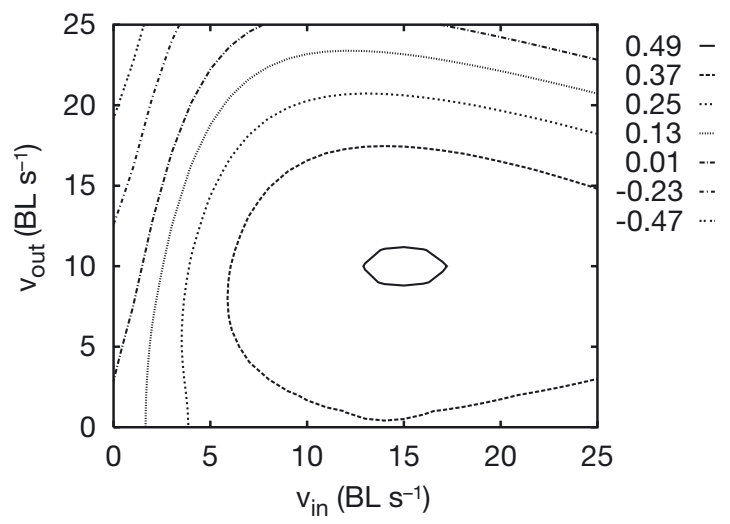

Fig. 5. Foraging efficiency in patchy environment with a 2-speed swimming strategy. Highest efficiency is reached by swimming at 15 body lengths $\mathrm{s}^{-1}$ inside patches and 10 body lengths $\mathrm{s}^{-1}$ outside patches. Key shows foraging efficiency $\left(\times 10^{6}\right)$ for each contour line

Fig. 4 shows the foraging efficiency for a larva swimming in a patchy environment. The environment is identical to the that in Fig. 3 in that the spatially averaged prey density is the same, but only $25 \%$ of the fluid volume is taken up with patches (resulting, therefore, in a 4 -fold increase in prey concentration within the patches relative to Fig. 3. As predicted earlier, these results are identical to those in Fig. 3. If the spatially averaged prey density is constant there is no difference in the predators foraging efficiency, provided the predator swims at a constant speed.

Fig. 5 shows the foraging efficiency for a larva swimming in a patchy environment with a strategy of swimming at different speeds in and out of patches, i.e. the results of plotting $F\left(V_{\text {in }}, V_{\text {out }}\right)$ as defined by Eq. (17). The optimal speeds are around $10 \mathrm{BL} \mathrm{s}^{-1}$ outside patches and $15 \mathrm{BL} \mathrm{s}^{-1}$ inside a patch. This gives a foraging efficiency around $7 \%$ higher than that reached in the constant-speed case. In other words, prey patchiness can be beneficial to a forager, provided it is able to detect patches and to optimise its swimming behaviour both within and outside patches. It is interesting to note that theory predicts that the larva should swim faster within patches, a prediction not entirely consistent with some observations, as discussed at more length in 'Discussion and conclusions'.

Fig. 6 shows an example of the 2-speed swimming strategy over a range of turbulence values. Turbulence strength, $\lambda$, is here defined by a minor modification of Eq. (4):

$$
W_{\mathrm{a}}=1.9 \lambda(\varepsilon a)^{1 / 3}
$$

This effectively splits the turbulent environment into 3 regions from the forager's point of view: low, medium and high turbulence. For low turbulence (approxi- mately $\lambda<2$ in Fig. 6) the optimal swimming behaviour is to swim at a finite speed both in and out of patches (cf. Fig. 5). For medium-strength turbulence $(2<\lambda<$ 100), the larvae should swim when inside a patch but not when outside a patch. For the highest turbulence regimes $(\lambda>100)$ the larvae should not swim at all. Numerical experiments show that this type of behaviour is structurally stable over a large range of valid parameter values.

The results in Fig. 6 make sense when one considers that the effects of turbulence are felt differently at different spatial scales; large-scale turbulence is of greater strength than small-scale turbulence (i.e. $L>$ $R)$. In a high-turbulence regime, $w_{L}$ is very large, and $W_{R}$ exceeds any reasonable swimming speed attainable by the larva; for a larva's swimming to make any appreciable difference to encounter rates, it must swim at a speed which is highly energetically unfavourable. In the medium turbulence regime $w_{L}$ is still large but $W_{R}$ is comparable to the predator's swimming speed (of the order of 1 to $10 \mathrm{BL} \mathrm{s}^{-1}$ ). Therefore when it comes to finding patches (i.e. $w_{L}$ is important), the larva's attempts are still insignificant compared to the turbulent effects, but inside patches the effort expended in swimming pays off in an increased local prey encounter rate, $\gamma$. Finally, in the lowest turbulence regime, both $w_{L}$ and $w_{R}$ are negligible and so turbulence offers little assistance to a predator seeking either patches or prey within patches. Put simply, the larvae are forced to do all the work.

These results are reflected in the absolute value of the optimal foraging efficiency, $F$; as turbulence increases so does $F$. This general conclusion will cease to be valid when one incorporates the fact that, in extremely high turbulence, only a fraction of the prey encountered are actually caught (MacKenzie \& Kiørboe 2000).

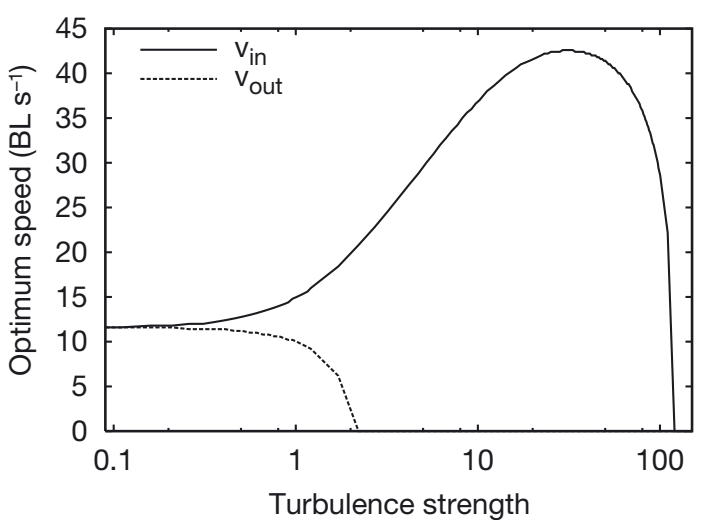

Fig. 6. Optimum swimming speeds in patchy environment for varying levels of turbulence. Turbulence strength $\lambda$ is as defined in Eq. (19), other parameters as in Table 1 


\section{PREDATORS FOLLOWING SIMPLE BEHAVIOURAL RULES}

Thus far the modelling has concentrated on the idealised case of a predator behaving 'optimally' in a given environment of prey and turbulence. In other words, although the predator is certainly 'small' in that its motions are strongly influenced by turbulence, it is behaving in a rather 'non-naïve' fashion. To assume that a fish larva is fully aware of its current global prey distribution, and of the current turbulent fluid motion, and is furthermore able to optimise nonlinear functions of 2 variables such as in Eq. (17), is perhaps unrealistic. It is the purpose of this section to investigate if, and how, a predator might forage in some near-optimal manner without perfect knowledge of its environment.

Since the predator is, for the purposes of this paper, only aware of the presence or absence of prey in a small hemispherical volume of radius $R$ centred on its eyes, any sensible behavioural rule must depend upon only this information and possibly upon some limited knowledge of the past. For example, rules could be based on simple assumptions; not having encountered prey for a certain amount of time might trigger increased swimming effort, or a lack of energy reserves might cause swimming to cease. Examples of how these simple rules could be incorporated in a foraging efficiency model, and a comparison of their relative benefits for the predator, form the basis of this section.

In order to incorporate dynamic changes of swimming speed based on past history, one must extend the basic model by allowing non-homogeneity in the renewal processes; the previously constant parameters $\alpha, \beta$ and $\gamma$ become functions of time, and should properly be regarded as stochastic processes themselves. When modelling the patchy case, this leads to a

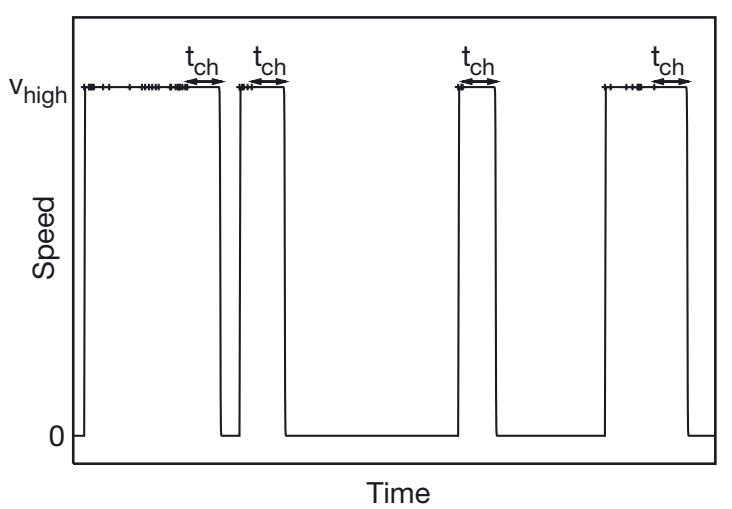

Fig. 7. Typical foraging history for predator following tanh function given by Eq. (20). Points show times when prey item is caught and cycle resets. $t_{\mathrm{ch}}$ (time until predator stops swimming) is also shown renewal process whose inter-arrival times depend on the inter-arrival times of a second renewal process (i.e. the length of time spent in a patch depends on how often the larva catches a prey within that patch).

The exact situation modelled below is one in which, when a predator encounters a prey, it immediately increases its speed to an upper limit. As the effect of eating wears off, the predator's swimming speed returns to its lower limit. This behaviour is modelled using a tanh function, although other functions, e.g. a step-down Heaviside function, could in principle be used. The choice of a (smooth) tanh function rather than a (discontinuous) Heaviside is a numerical expedient, facilitating the optimisation of the foraging efficiency function, and also allowing some analytical progress to be made, although only in rather limited special cases not presented here. Explicitly, the rule is:

$$
v(t)=\frac{v_{\text {high }}\left(\tanh \left(t_{\mathrm{ch}}-t\right)+1\right)}{2}
$$

as illustrated in Fig. 7. This implies the lower swimming speed is zero, chosen because this is the optimal swimming speed outside patches for moderate to high levels of turbulence (see Fig. 6). This may not be an appropriate formulation for low-turbulence environments, where it is advantageous and indeed necessary for the predator to swim between patches; other behavioural rules could readily be incorporated into the model, without additional conceptual difficulty, to investigate such scenarios. On catching a prey item, the larva swims at speed $v_{\text {high }}$ until time $t_{\mathrm{ch}}$, when it effectively stops swimming. Every time a prey item is caught the speed increases to $V_{\text {high }}$ and the clock is reset back to zero. Fig. 7 illustrates how this behavioural rule would effect the larvae's speed over a period of time. In a non-patchy environment it is possible to calculate the foraging efficiency using results from the theory of non-homogeneous Poisson processes. However in a patchy environment it has not proved possible to derive closed-form expressions for $F$, and the results presented below are obtained from IBM simulations. The IBM simulations calculate the mean efficiency of a larva over several thousand foraging bouts using an explicit Euler method.

Fig. 8 shows the effect of altering the length of time for which the higher speed behaviour is employed i.e. of varying $t_{\mathrm{ch}}$ in Eq. (20). The lower curves are for the default patchy scenario summarised by the parameters in Table 1. The graph shows 2 different values of $v_{\text {highi }}$ the first is $V_{\text {high }}=25 \mathrm{BL} \mathrm{s}^{-1}$, the second $v_{\text {high }}=10 \mathrm{BL} \mathrm{s}^{-1}$, the optimum constant speed for this environment. Note that a very large value of $t_{\mathrm{ch}}$ is equivalent to swimming at a constant speed $\left(V_{\text {high }}\right)$ all the time, and that as $t_{\mathrm{ch}}$ decreases toward zero the larva is effectively ceasing to swim. The higher curves show the same scenario, 


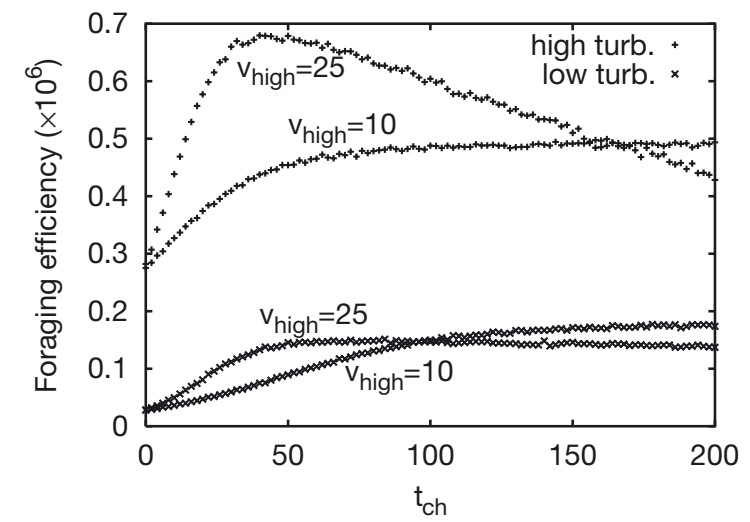

Fig. 8. How variations in the tanh function Eq. (20) affect efficiency of a larva following simple behavioural rule in a patchy environment. Foraging efficiency is plotted against the $t_{\mathrm{ch}}$ parameter in Eq. (20). Lower curves show larva in low-turbulence environment (all parameters as in Table 1); higher curves represent higher turbulence environment (all parameters as in Table 1, except turbulent velocities, which are 10 times original values). For both levels of turbulence 2 values of $v_{\text {high }}$ are plotted

but here the turbulent velocities have been increased by a factor of 10 . In the lower turbulence environments this behavioural function appears to be of little use to the predator, indeed it seems that the optimal behaviour is to pick a moderate value of $v_{\text {high }}$ and then to allow $t_{\mathrm{ch}}$ to tend to infinity, i.e. reverting back to the model for heterogeneous (patchy) prey at constant swimming speed. However, it is clear that in a higher turbulence environment this is not the case and it is more beneficial to the predator to swim at a higher speed for a short amount of time.

Fig. 9 compares the success of different behavioural strategies for larvae employing 1- or 2-speed strategies, at low levels of turbulence. The constant case shows the foraging efficiency of a larva swimming at speed $v$ between 0 and $20 \mathrm{BL} \mathrm{s}^{-1}$; the optimum speed is approximately $11 \mathrm{BL} \mathrm{s}^{-1}$ (cf. Fig. 4). The 2-speed case shows a larva swimming at $10 \mathrm{BL} \mathrm{s}^{-1}$ outside the patches and variable speed $v$ inside patches; here the optimum speed inside a patch is $15 \mathrm{BL} \mathrm{s}^{-1}$ (cf. Fig. 5). The 2-speed forager has a slightly higher optimum efficiency than the constant-speed forager, but whether (given the limitations of the model) this difference is significant biologically is open to debate. The behavioural rule (Eq. 20) is implemented for a larva swimming at the indicated speed ( 0 to $25 \mathrm{BL} \mathrm{s}^{-1}$ ) for $100 \mathrm{~s}$ after each prey encounter before ceasing to swim. The results are robust to other choices of $t_{\mathrm{ch}}$ around the optimal values suggested by Fig. 8 . The key result illustrated by Fig. 9 is that the larva following a behavioural rule has a foraging efficiency up to 1 order of magnitude less than that of 'omniscient'

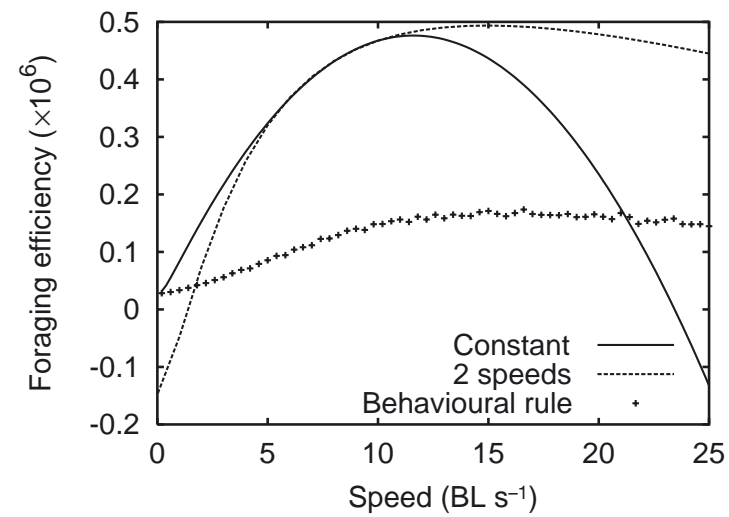

Fig. 9. Comparison of different behavioural strategies in lowturbulence regime (all parameters as in Table 1)

larvae. This finding is not unique to the parameter values chosen; all the numerical investigations carried out show that the larva following simple local rules fares badly in this low-turbulence regime.

Fig. 10 shows the outcomes of the same strategies in a high-turbulence environment (the turbulent velocities, $w_{L}$ and $w_{R}$ are increased by a factor of 10 ). In this scenario, the omniscient 2 -speed predator still attains the greatest foraging efficiency, but here the singlespeed forager and behavioural rule forager fare similarly poorly, with the single-speed foreager faring worst of all. Again, this general result is robust to reasonable changes in the biological parameter values chosen.

Other behavioural rules can be, and have been, the subject of similar numerical investigations. For example, one could reverse the rule of Eq. (20) to simulate a larva swimming at a constant high speed until encountering a prey, when its speed drops for some fixed duration before the higher speed is resumed. How-

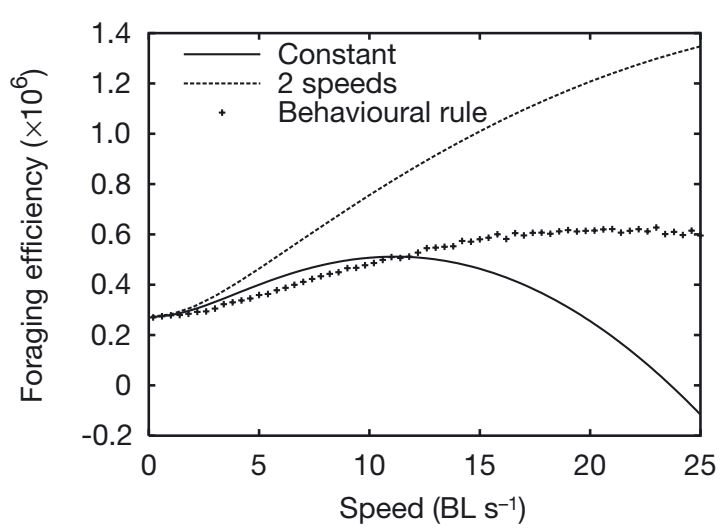

Fig. 10. Comparison of different behavioural strategies in high-turbulence regime (all parameters as in Table 1, except turbulent velocities, which are 10 times original values) 
ever, none of the rules of this nature investigated by the authors give foraging efficiencies comparable to those attained by omniscient predators whose swimming speed can alter due to the presence or absence of a patch. The above results do not depend on the choice of a tanh function in Eq. (20); numerical simulations using an approximately equivalent Heaviside function produce qualitatively identical and quantitatively indistinguishable results.

The only possible advantage of following a behavioural rule to emerge from the numerical investigations is that a wide range of parameters (e.g. $t_{\mathrm{ch}}$ and $v_{\text {high }}$ in Eq. 20) result in a positive foraging efficiency. This means that larvae following such a strategy do not need to choose a 'perfect' rule to follow to achieve a near-optimal foraging efficiency. In contrast, if a larva swimming at a constant speed or following omniscient behaviour chooses the wrong swimming speed, then the consequences for foraging efficiency can be disastrous. A larva following a behavioural rule has much more flexibility to swim at a non-optimum speed and still have a reasonable efficiency. However, the efficiencies obtained by such a larva never come close to those for optimal omniscient larvae, and so this finding may be of limited practical relevance.

\section{DISCUSSION AND CONCLUSIONS}

It is useful to discuss the predictions of the very simple model presented above in the context of existing experimental data. Some general results from the model are in full agreement with observations, but others seem to be contradicted by data, and it is the belief of the authors that these apparent contradictions are of some importance.

The main result, summarised graphically in Figs. 2 $\& 6$, is that in general one expects swimming activity to decrease as turbulence increases. This is entirely consistent with observations; for example, studies by MacKenzie \& Kiørboe (1995) and Utne-Palm \& Stiansen (2002) demonstrated that herring larvae swim significantly less in more turbulent water compared to still water.

The homogeneous model also predicts that the predator should respond to an increase in prey concentration by increasing its swimming speed (see 'Homogeneous prey distribution, constant swimming speed'). In an experimental scenario this result would become manifest by observing larvae swimming faster in treatments with higher prey densities. In contrast to this prediction, Døving et al. (1994) showed that predators respond to an increase in arganine concentration (used as a proxy for prey concentration) by reducing their swimming activity and by swimming more slowly.
Similarly, Munk (1995) demonstrated that larvae spend less time swimming as prey density increases. A common factor in these studies was that they were carried out in a non-turbulent environment. The heterogenous model predicts that, in a non-turbulent environment with zero handling time, predators should swim at the same speed inside and outside a patch; numerical investigations show that these results hold for non-zero handling times. However, this ceases to be true when even small amounts of turbulence are introduced; the optimal strategy is to expend less swimming effort outside patches.

It is possible that the experimentally observed larvae are showing behaviour adapted to a homogeneous, rather than a patchy, environment. In this case the observations agree with the model's predictions. However, the results depicted in Figs. 9 \& 10 indicate strongly that a predator which chooses a single-speed strategy (i.e. one that disregards patchiness) can be at a significant disadvantage compared to a predator which can respond to, and exploit, prey heterogeneity. Since the copepod prey species of larval fishes are well known to exhibit strong spatial patchiness at spatial scales ranging from metres to 10 s of kilometres (Tsuda et al. 1993, Currie et al. 1998, Tokarev et al. 1998) it seems likely that such an explanation is correct.

In summary, it is vital to ascertain whether larvae observed in an artificial laboratory environment are behaving appropriately to that environment, or are displaying behaviour the advantage of which only becomes clear when considered in the context of natural environmental conditions. This increases the need for careful experimental effort concerning the behaviour of foragers in differing levels of prey density and turbulence. It should also be mentioned that the studies of Døving et al. (1994) and Munk (1995) involved cod, and not herring, larvae. Cod employ a slightly different foraging strategy to herring, using short bursts of motion followed by pauses rather than the more continuous cruising of herring. Adapting the model to allow for the saltatory behaviour of cod may be an interesting source of future research, but is not addressed herein.

The following conclusions apply to any predator foraging at random in a turbulent environment, subject to the basic model assumptions:

- The optimal swimming speed decreases as turbulence increases.

- Above some critical level of turbulence, the optimal strategy is to cease swimming altogether.

- When the prey is patchily distributed, the predator should behave so as to swim more slowly outside patches. There are turbulent regimes in which the optimal strategy is to swim only while within patches. 
- A predator with only local knowledge of its surroundings, and with behavioural rules based on its recent foraging history, will be at a significant evolutionary disadvantage compared to a rival predator with a more global knowledge of its environment.

A further important conclusion concerning the evolution of behaviour is less easily quantified but is demonstrated by Figs. $9 \&$ 10. Predators which cannot respond to local prey conditions, or which are able to respond according to local rules but which are unaware of the global environment, fare very badly in comparison to 'omniscient' predators. In other words, 'small and naïve' predators within a turbulent and patchy environment are subject to strong selective pressures to evolve forms of global 'intelligence'. Such a global awareness could be achieved by developing an increased visual range so as to accurately identify the presence, and prey density, of a patch. The fact that the value of omniscience is particularly significant in relatively low-turbulence regimes (Fig. 9), where prey-generated chemical signals within the fluid are less likely to be corrupted by fluid movement, suggests that the role of oflaction may play a crucial role in governing predator behaviour, and this forms the subject of ongoing theoretical research. It should be noted that the above model does not take into account any additional metabolic costs required for development and maintenance of improved sensory organs; such factors must be taken into account in any more quantified discussion of evolution and selective pressure.

Appendix 1. Calculation of foraging efficiencies

Foraging efficiency is defined as the expected gain in energy over a foraging cycle, divided by the expected time taken for that cycle:

$$
F=\frac{E(\Delta G)}{E(\Delta t)}
$$

However, one must be precise about what one means by a foraging cycle. For the homogeneously distributed prey and constant-speed-predator case, a cycle is defined as the time taken to find and consume 1 prey [Fig. 1a]), so that:

$$
\begin{gathered}
\Delta G=Q-D_{0} V^{2} t^{*} \\
\Delta t=t^{*}+\tau
\end{gathered}
$$

where the random variable $t^{*}$ is the time of first arrival of a Poisson process with intensity $\gamma$ and therefore has the probability density function:

$$
f\left(t^{*}\right)=\gamma \mathrm{e}^{-\gamma t^{*}}
$$

Hence

$$
\begin{aligned}
E(\Delta G) & =\int_{0}^{\infty} \Delta G f\left(t^{*}\right) d t^{*} \\
E(\Delta t) & =\int_{0}^{\infty} \Delta t f\left(t^{*}\right) d t^{*}
\end{aligned}
$$

giving the foraging efficiency function $F$ to be:

$$
F=\frac{E(\Delta G)}{E(\Delta t)}=\frac{Q-\frac{D_{0} v^{2}}{\gamma}}{\frac{1}{\gamma}+\tau}=\frac{Q \gamma-D_{0} v^{2}}{1+\gamma \tau}
$$

This agrees with Eqs. (5) \& (7) for the special (homogeneous prey) case where $\beta=0$ and the $\alpha$ s cancel.

In the heterogeneous case the argument is less straightforward. An appropriate choice of foraging cycle is one which covers the (stochastic) time interval from leaving one patch until leaving the next (as indicated in Fig. 1b). The time taken to find a patch, $t_{\text {out }}$ is the time of first arrival of the Poisson process with rate $\alpha$ and the energy used whilst swimming to find a patch is given by:

$$
\Delta G_{\text {out }}=-D_{0} v^{2} t_{\text {out }}
$$

Similarly, the time spent in a patch, $t_{\mathrm{in}}$, is given by the time of first arrival of a Poisson process with intensity $\beta$. Whilst within the patch, the larva will forage as in the homogeneous case until it reaches time $t_{\text {in }}$. Define a random variable $N$ to be the number of prey caught whilst in a patch. The energy change whilst in a patch is then given by:

$$
\Delta G_{\text {in }}=N Q-D_{0} V^{2} t_{\text {in }}
$$

The total time for a whole foraging cycle (as illustrated in Fig. 1b) is:

$$
\Delta t=t_{\text {out }}+t_{\text {in }}+N \tau
$$

The predator spends $t_{\text {out }}$ and $t_{\text {in }}$ swimming outside and within the patch respectively, plus an extra time $N \tau$ in capturing encountered prey. This gives the expected value of the foraging efficiency as:

$$
\begin{aligned}
F & =\frac{E(\Delta G)}{E(\Delta t)}=\frac{E\left(\Delta G_{\text {out }}\right)+E\left(\Delta G_{\text {in }}\right)}{E\left(t_{\text {out }}\right)+E\left(t_{\text {in }}\right)+E(N \tau)} \\
& =\frac{-D_{0} v^{2} E\left(t_{\text {out }}\right)+Q E(N)-D_{0} v^{2} E\left(t_{\text {in }}\right)}{E\left(t_{\text {out }}\right)+E\left(t_{\text {in }}\right)+\tau E(N)}
\end{aligned}
$$

where $E(N)$, the expected value of the number of prey caught in a single visit to a patch, is given by the average of number of arrivals of the Poisson process governed by rate $\gamma$ before the first arrival of the Poisson process governed by rate $\beta$.

Using results from Pitchford \& Brindley (2001), viz:

$$
E(N)=\frac{E\left(t_{\text {in }}\right)}{E\left(t^{*}\right)}=\frac{\gamma}{\beta}
$$

the foraging efficiency is then given by:

$$
F=\frac{Q \gamma \alpha-D_{0} V^{2}(\alpha+\beta)}{(\alpha+\beta+\alpha \gamma \tau)}
$$

which is, after substitution from Eq. (5), identical to Eq. (7). 
Acknowledgements. We are grateful to 3 anonymous reviewers whose insightful comments and criticisms have greatly improved this article.

\section{LITERATURE CITED}

Beyer JE, Nielsen BF (1996) Predator foraging in patchy environments: the interrupted Poisson process (IPP) model unit. Dana 11:65-130

Charnov EL (1976) Optimal foraging, the marginal value theorem. Theor Popul Biol 9:129-136

Cox DR (1962) Renewal theory. In: Monographs on applied probability and statistics. Methuen, London

Currie WJS, Claereboudt MR, Roff JC (1998) Gaps and patches in the ocean: a one-dimensional analysis of planktonic distributions. Mar Ecol Prog Ser 171:15-21

Cushing DH, Horwood JW (1994) The growth and death of fish larvae. J Plankton Res 16:291-300

Dabrowski KR, Kok LY, Takashima F (1986) How efficiently do fish larvae and juveniles swim? Comp Biochem Physiol A 85:657-661

Dabrowski K, Takashima F, Law Y (1988) Bioenergetic model of planktivorous fish feeding, growth and metabolism: theoretical optimum swimming speed of fish larvae. J Fish Biol 32:443-458

Døving KB, Mårstøl M, Andersen JR, Knutsen JA (1994) Experimental evidence of chemokinesis in newly hatched cod larvae (Gadus morhua L.). Mar Biol 120:351-358

Fuiman L, Batty R (1997) What a drag it is getting cold: partitioning the physical and physiological effects of temperature on fish swimming. J Exp Biol 200:1745-1755

Gallego A (1994) Changes in the swimming behaviour of larval herring in response to two different prey densities. J Mar Biol Assoc UK 74:955-958

Hill PS, Nowell ARM, Jumars PA (1992) Encounter rate by turbulent shear of particles similar in diameter to the Kolmogorov scale. J Mar Resh 50:643-668

Houston AI, McNamara JM (1999) Models of adaptive behaviour. Cambridge University Press, Cambridge

Houston AI, McNamara JM (2001) Optimality models in behavioral biology. SIAM (Soc Ind Appl Math) Rev 43:413-466

Editorial responsibility: Thomas Kiørboe (Contributing Editor), Charlottenlund, Denmark
Lewis D, Pedley T (2001) The influence of turbulence on plankton predation strategies. J Theor Biol 210:347-365

MacKenzie B, Kiørboe T (1995) Encounter rates and swimming behavior of pause-travel and cruise larval fish predators in calm and turbulent laboratory environments. Limnol Oceanogr 40(7):1278-1289

MacKenzie B, Kiørboe T (2000) Larval fish feeding and turbulence: a case for the downside. Limnol Oceanogr 45: $1-10$

Munk P (1995) Foraging behaviour of larval cod (Gadus morhua) influenced by prey density and hunger. Mar Biol 122:205-212

Ollason (1980) Learning to forage-optimally. Theor Popul Biol 18:44-56

Pitchford J, Brindley J (2001) Prey patchiness, predator survival and fish recruitment. Bull Math Biol 63:527-546

Rothschild BJ, Osborn TR (1988) Small scale turbulence and plankton contact rates. J Plankton Res 10:465-474

Stephens DW, Krebs JR (1986) Foraging theory. Princeton University Press, Princeton, NJ

Sundby S (1997) Turbulence and ichthyoplankton: influence on vertical distributions and encounter rates. Sci Mar 61 (Suppl 1):159-176

Templeton A, Lawlor L (1981) The fallacy of the averages in ecological optimization theory. Am Nat 117:390-391

Tokarev YN, Williams R, Piontkovski SA (1998) Small-scale plankton patchiness in the Black Sea euphotic layer. Hydrobiologia 375/376:363-367

Tsuda A, Sugisaki H, Ishimaru T, Saino T, Sato T (1993) White-noise-like distribution of the oceanic copepod Neocalanus cristatus in the subarctic North Pacific. Mar Ecol Prog Ser 97:39-46

Utne-Palm AC, Stiansen JE (2002) Effect of larval ontogeny, turbulence and light on prey attack rate and swimming activity in herring larvae. J Exp Mar Biol Ecol 268: $147-170$

Visser AW, Mackenzie BR (1998) Turbulence-induced contact rates of plankton: the question of scale. Mar Ecol Prog Ser 166:307-310

Weiser W, Kaufmann R (1998) A note on interactions between temperature, viscosity, body size and swimming energetics in fish larvae. J Exp Biol 201:1369-1372

Submitted: June 21, 2002; Accepted: January 6, 2003

Proofs received from author(s): June 17, 2003 